\title{
Self Assembling Peptide Nanofibers for Extracellular Matrix Remodeling in Diabetic Cardiomyopathy
}

Jennifer R. Hurley¹, Abdul Q. Sheikh¹, Wei Huang², Yigang Wang ${ }^{2}$ and Daria A. Narmoneva1*

${ }^{1}$ School of Energy, Environmental, Biological \& Medical Engineering, University of Cincinnati, Cincinnati, Ohio, USA ${ }^{2}$ Department of Pathology and Laboratory Medicine, University of Cincinnati Medical Center, Cincinnati, Ohio, USA

\begin{abstract}
In the diabetic heart, increased collagen accumulation, stiffness and cardiac dysfunction may be linked to the reduced expression and activity of matrix metalloproteinase-2 (MMP-2), suggesting that diabetes-associated cardiac fibrosis may be attenuated through stimulation of native MMP-2 expression or delivery of exogenous MMP-2. Peptide nanofibers were investigated as a microenvironment for cardiac regeneration via endogenous MMP-2 stimulation or exogenous MMP-2 delivery to promote matrix remodeling by wild type and diabetic cardiac fibroblasts. Cells were isolated from wild type or diabetic rat hearts and embedded in nanofibers, nanofibers with exogenous MMP-2, and Matrigel controls for 1,6 and 14 days. Responses associated with matrix remodeling were assessed, including cell survival, native MMP-2 expression, ECM deposition and construct stiffness. The results demonstrate that nanofiber scaffolds provide an effective delivery vehicle with gradual MMP-2 release, while supporting long term survival and temporal matrix remodeling by cardiac fibroblasts. Nanofiber scaffolds maintained the balance between cell proliferation and apoptosis, in contrast to increased apoptosis with time in culture in Matrigel. Diabetic and wild type fibroblasts showed different temporal trends for MMP-2 expression, collagen I deposition and scaffold stiffness, indicating increased matrix remodeling by diabetic cells in the nanofiber microenvironment. The data suggest that stimulation of native MMP expression by the NFs alone may be the more Suitable strategy to improve reparative matrix remodeling. Overall, the results suggest that peptide nanofibers may be uniquely suited to increase local MMP-2 concentration in the diabetic heart and may be promising for applications focused on therapeutic matrix remodeling and cardiac regeneration.
\end{abstract}

Keywords: Peptide nanofibers; Cardiac fibroblasts; MMP-2; Protein delivery; Extracellular matrix remodeling; Cardiac regeneration; Diabetes; Diabetic cardiomyopathy

\section{Introduction}

Diabetes is one of the most common chronic illnesses in the world and 25.8 million individuals $-8.3 \%$ of the population- are afflicted in the United States alone with annual medical costs estimated at $\$ 174$ billion in 2007 [1]. While there are many life-threatening complications associated with diabetes, heart disease is one of the most severe with a higher than normal diabetes-associated death rate. Diabetic cardiomyopathy (DCM) is a diabetes-associated cardiovascular condition defined as ventricular dysfunction in the absence of other etiological factors, such as hypertension or coronary heart disease [2-4], which results in pathological alterations to the myocardium including circulatory defects, impaired heart muscle contraction, and progressive fibrosis. The elusive and poorly defined nature of DCM indicates that there exists a need for novel approaches for treatment of diabetic cardiomyopathy which may focus on alternative molecular mechanisms for the disease. Extracellular matrix (ECM) turnover and remodeling are essential in many physiological processes yet their regulation is impaired in DCM, leading to damaging structural, geometric and functional changes in the heart $[2,3,5,6]$. ECM turnover can be regulated by many factors, including matrix metalloproteinases (MMPs) [6], angiotensin II [7], aldosterone [4], nuclear factor kappa $\mathrm{B}(\mathrm{NF}-\kappa \mathrm{B})$ [8], transforming growth factor-beta 1 (TGF- $\beta 1$ ) [9], nitric oxide [10], advanced glycation end products [5], and kinins [11]. Importantly, recent studies of diabetic human patients [12,13] and in animal models $[7,9,14-16]$ suggest that MMP activity is impaired in diabetes, thus highlighting this particular mechanism as a novel therapeutic target. In particular, studies such as these have shown that dysregulation of cardiac MMP-2 expression contributes to the increased collagen deposition, progressive fibrosis, increased ventricular stiffness, and cardiac dysfunction seen in diabetic cardiomyopathy in rodent animal models. This MMP-2 deficiency likely stems from diabetes-related changes to the cardiac fibroblast phenotype, such as increased collagen synthesis by cardiac fibroblasts under diabetic conditions [17-19]. At the same time, studies in murine models of type II ( $\mathrm{db} / \mathrm{db})$ diabetes have shown decreased MMP activation in diabetic fibroblasts, as well as impairments in vital cellular processes, including reduced growth factor expression and reduced cellular migration [20] Therefore, overcoming the inhibitory effects of diabetic conditions on matrix remodeling by cardiac fibroblasts through stimulation of native MMP-2 expression or delivery of exogenous MMP-2 represents a novel target for therapeutic treatment of DCM.

Peptide nanofibers, such as RAD16-II, represent a scaffold-based tissue engineering approach which can be effectively used for local and controlled delivery of proteins to the myocardium $[21,22]$ to promote cardiac regeneration via matrix remodeling. RAD16-II nanofibers are made via the spontaneous assembly of self-complementary oligopeptides consisting of alternating hydrophilic and hydrophobic

*Corresponding author: Daria A. Narmoneva, Ph.D., School of Energy Environmental, Biological \& Medical Engineering, University of Cincinnati, 601 Engineering Research Center 2901 Woodside Drive, Cincinnati, OH 45221-0048, Tel: 513-556-3997; Fax: 513-566-4612; E-mail: daria.narmoneva@uc.edu

Received November 08, 2011; Accepted December 15, 2011; Published December 30, 2011

Citation: Hurley JR, Sheikh AQ, Huang W, Wang Y, Narmoneva DA, et al. (2012) Self Assembling Peptide Nanofibers for Extracellular Matrix Remodeling in Diabetic Cardiomyopathy. J Bioengineer \& Biomedical Sci S5:003. doi:10.4172/2155-9538. S5-003

Copyright: @ 2012 Hurley JR, et al. This is an open-access article distributed unde the terms of the Creative Commons Attribution License, which permits unrestricted use, distribution, and reproduction in any medium, provided the original author and source are credited. 
amino acids [23]. The material and biochemical properties of the nanofibers can be easily tailored via sequence alterations and peptide concentration. RAD16-II and similar nanofibers have been extensively studied in vitro for controlled protein delivery [24,25]. Additionally, these nanofibers have been used in vivo for temporally-controlled and localized cardiac delivery of proteins, including growth factors IGF1 [21], PDGF-BB [24], and SDF-1 [22]. Importantly, nanofiber-based scaffolds represent a potentially very attractive proteolytically-stable microenvironment suitable for MMP delivery, in contrast to native ECM-based materials which are rapidly degraded by the proteases expressed by the cells or present in the extracellular environment. Our previous studies have demonstrated that RAD16-II peptide nanofibers significantly enhance native expression of MMP-2 by human dermal fibroblasts as compared with collagen I controls, while maintaining long-term cell survival and scaffold stability [26]. The goal of this study was to determine if self-assembling peptide nanofibers could be used to create a proteolytically stable extracellular microenvironment for long-term MMP delivery and enhancement of cardiac remodeling. This study tested the hypothesis that increased MMP-2 concentration, either native or exogenous, in the nanofiber microenvironment would promote matrix remodeling by diabetic cardiac fibroblasts in vitro. Our results suggest that a nanofiber-based approach may be a promising cardiac tissue engineering strategy to stimulate reparative extracellular matrix remodeling in the diabetic heart.

\section{Materials and Methods}

\section{MMP-2/NF delivery system}

To quantify protein release in vitro from peptide nanofibers (NFs), active human MMP-2 was incorporated into the NFs. Human MMP2 was selected in order to distinguish it from the native rat MMP-2 expressedbyfibroblastsin subsequentexperiments. Activehuman MMP2 (EMD Chemicals, Gibbstown, NJ) was incorporated into nanofibers (RAD16-II, (RARADADA) $)_{2}, 1.0 \%$ w/w, SynBioSci, Livermore, CA) via non-covalent binding at $100 \mathrm{ng} / \mathrm{ml}$. This concentration was selected as it falls well below the peptide nanofibers protein binding capacity $(\sim 1.0 \mathrm{ng}$ protein/ $\mu \mathrm{g}$ peptide [24]) and is similar to normal cardiac MMP- 2 levels $[27,28]$. Nanofibers without MMP-2 and Matrigel (BD Biosciences, Bedford, MA) served as controls. $75 \mu$ l of scaffold solution was added to culture inserts and incubated in phosphate-buffered saline (PBS) at $37^{\circ} \mathrm{C}$ to form three-dimensional scaffolds. Supernatant (PBS after incubation) was collected and replaced at 0.5, 1, 2, 4, 6, 12, 24, 36, 48, and 72 hrs. Supernatant samples were tested using Human MMP-2 Quantikine ELISA Kit (R\&D Systems, Minneapolis, MN) to determine MMP-2 release kinetics from NFs.

\section{Streptozotocin type I diabetes rat model}

All animal procedures were performed using protocols approved by the University of Cincinnati Institutional Animal Care and Use Committee. Diabetic (db) and wild type (wt) cardiac fibroblasts were isolated from 12-16 week old female Sprague Dawley rats (SAS SD Strain 400, Charles River, Wilmington, MA). Type I diabetes was induced in 8 week old rats using a single intraperitoneal injection of streptozotocin (70 mg/kg, Sigma-Aldrich, St. Louis, MO) [29]. The streptozotocin rat model was chosen as it closely mimics the timedependent disease progression of diabetic cardiomyopathy [30]. Immediate onset of diabetes was confirmed with serum glucose levels $>450 \mathrm{mg} / \mathrm{dl}$. Six weeks after injection, diabetic animals and age and strain-matched wild type controls were sacrificed and heart tissue was harvested either for cardiac cell isolation or embedded in paraffin for histological analysis.

\section{Picrosirius red staining}

Sections of heart tissue were harvested, fixed in $10 \%$ formalin paraffin embedded, and sectioned at $5 \mu \mathrm{m}$ thickness. To assess cardiac fibrosis, fibrillar collagen content was measured using picrosirius red staining of cardiac tissue sections, imaged under polarized light [31].

\section{Cell isolation}

For cell isolation, heart tissue were harvested and washed in cold PBS several times, finely chopped and digested first in trypsin (1 mg/ $\mathrm{ml}$, Sigma-Aldrich, St. Louis, MO) at room temperature for 1 hour and then collagenase I $(172 \mathrm{U} / \mathrm{ml}$, Worthington Biochemical Corp., Lakewood, NJ) at $37^{\circ} \mathrm{C}$ for 45 minutes. Supernatant containing cells was separated from remaining tissue and centrifuged. The cell pellet was resuspended and added to uncoated cell culture dishes for 2 hours to allow for attachment and cultured in Medium 199 (HyClone, Logan, UT) containing $10 \%$ fetal bovine serum (FBS; Atlanta Biologicals, Lawrenceville, GA), 1\% Antibiotic-Antimycotic (Atlanta Biologicals, Lawrenceville, GA), $10 \mu \mathrm{g} / \mathrm{ml}$ heparin (Sigma-Aldrich, St. Louis, MO), and $0.2 \mathrm{ng} / \mathrm{mL}$ cell growth supplement (Sigma-Aldrich, St. Louis, MO) as previously described $[26,32]$. Cell cultures were maintained at $37^{\circ} \mathrm{C}$ in $100 \%$ humidified air containing $5 \% \mathrm{CO}_{2}$. Cells of passage $4-10$ were used in all experiments.

\section{Sample preparation}

To quantify the effects of peptide nanofibers (NFs) on native MMP release and matrix remodeling by wild type (wt) and diabetic (db) cardiac fibroblasts, a total of six experimental groups were created: NF, NF+MMP, Matrigel, each with wt or db cells. Active human MMP-2 was incorporated into the NFs for exogenous protein delivery in order to distinguish it from the native rat MMP-2 expressed by the cells. Matrigel was selected as a control three-dimensional microenvironment as it does not induce MMP-2 expression and activation in vitro [33,34].

Diabetic and wild type fibroblasts (passages 4-10) were three-dimensionally embedded in nanofibers (NF, RAD16-II, (RARADADA), $1.0 \% \mathrm{w} / \mathrm{w}$, SynBioSci, Livermore, CA), NFs+100 ng/ $\mathrm{ml}$ active human MMP-2 (NF+MMP, EMD Chemicals, Gibbstown, NJ), and Matrigel (BD Biosciences, Bedford, MA) at a density of $2.5 \times 10^{6}$ cells $/ \mathrm{ml}$ (protein expression, rheometry, and cell proliferation) or surface seeded at $1.0 \times 10^{4}$ cells/insert (cell apoptosis). Scaffolds without cells served as controls for protein expression and rheometry experiments. Samples were cultured for 1, 6 and 14 days with daily media changes. For all ELISA experiments, cell culture medium (M199 with $10 \%$ FBS, $1 \%$ Antibiotic-Antimycotic, and $10 \mu \mathrm{g} / \mathrm{ml}$ heparin) without growth supplement was used with daily medium changes.

\section{Cell phenotype}

Staining was performed to confirm fibroblast phenotype of the primary cells and for visualization of cells within the three-dimensional scaffolds. Wild type and diabetic fibroblasts were either cultured in gelatin-coated wells in 24-well tissue culture plates until near confluency or embedded in NF, NF+MMP and Matrigel scaffolds at a density of $2.5 \times 10^{6}$ cells $/ \mathrm{ml}$ and cultured with daily media changes for 1,6 and 14 days. Samples were fixed with methanol/acetone. Samples were stained with vimentin (a fibroblast marker [35]) and a-smooth muscle actin ( $\alpha$-SMA, a marker of myofibroblasts [36]) primary antibodies (Sigma-Aldrich, St. Louis, MO) and appropriate fluorescent secondary antibodies (Alexa Fluor ${ }^{\oplus}$, Invitrogen Corp., Carlsbad, CA) and DAPI (Invitrogen Corp., Carlsbad, CA). Imaging of stained cells was performed with an inverted fluorescent microscope (Olympus IX81) and positive stained cells were counted (3 images/sample). 
Citation: Hurley JR, Sheikh AQ, Huang W, Wang Y, Narmoneva DA, et al. (2012) Self Assembling Peptide Nanofibers for Extracellular Matrix Remodeling in Diabetic Cardiomyopathy. J Bioengineer \& Biomedical Sci S5:003. doi:10.4172/2155-9538.S5-003

\section{Cell apoptosis}

LIVE/DEAD ${ }^{\circledR}$ Kit (Molecular Probes, Carlsbad, CA) was used to assess cell apoptosis at 1,6 and 14 days in culture. The kit was used per manufacturer instructions with 30 minute incubation with Calcein AM and Ethidium homodimer-1 followed by imaging of stained cells with an inverted fluorescent microscope (Olympus IX81). Total numbers of live and dead cells were counted (3 images/sample) to determine percentage of apoptotic cells.

\section{Cell proliferation}

CellTiter $96^{\circ}$ Aqueous non-radioactive cell proliferation assay (Promega Corporation, Madison, WI) was used to assess cell proliferation at 1,6 , and 14 days in culture. Cells were embedded in scaffolds at a density of $2.5 \times 10^{6}$ cells $/ \mathrm{ml}$ and cultured with daily medium changes. At each time point, samples were incubated in medium containing MTS/PMS solution for 3 hours per manufacturer instructions. Media samples from culture inserts were placed in a 96well plate and absorbance was measured at 490nm using an ELISA plate reader. All data were normalized to day 1 values for analyses. After testing, MTS/PMS medium was aspirated and fresh medium was added to the samples.

\section{Sample total protein content determination}

Cell-scaffold constructs were cultured in no growth factor medium (cell culture medium without additional growth factor supplementation) and collected at days 1,6 and 14. Samples were stored in TriReagent (Molecular Research Center, Cincinnati, $\mathrm{OH}$ ) at $-80^{\circ} \mathrm{C}$ until testing. Protein isolation was performed per the manufacturer's protocol. Total protein content in the samples was determined using Coomassie Plus Assay Kit (Thermo Fisher Scientific, Rockford, IL).

\section{Protein expression using Enzyme-Linked Immunosorbent Assay (ELISA)}

Cell-scaffold constructs were cultured in no growth factor medium (cell culture medium without additional growth factor supplementation) and media and matrix samples were collected at days 1,6 and 14 and stored at $-80^{\circ} \mathrm{C}$ until testing. ELISAs were performed as described in [37] to determine protein concentrations in medium samples (Rat MMP-2 - antibodies from R\&D Systems, Minneapolis, $\mathrm{MN}$ ) and matrix samples (Rat MMP-2, Rat Collagen I - antibodies from Pierce Biotechnology, Rockford, IL, and Rat Collagen IV - antibodies from Abcam, Cambridge, MA). Protein expression in matrix samples was normalized using total protein content. For all ELISAs, additional controls of the cell culture medium alone (containing 10\% serum) and noncellular scaffolds were included to confirm that protein content in both the serum and scaffold would not affect protein expression and detection, with no differences observed between control samples and the $0 \mathrm{pg} / \mathrm{ml}$ standard.

\section{Mechanical testing of cell-scaffold constructs using rheometry}

Elastic moduli (G') of NF, NF+MMP and Matrigel with living cells or scaffolds alone (controls) at 1,6, and 14 days were measured with a parallel-plate rheometer (Bohlin Instruments Inc., East Brunswick, NJ) as described previously [26]. Briefly, circular constructs of $8 \mathrm{~mm}$ diameter and approximately $500 \mu \mathrm{m}$ height were formed on glass slides using molds, covered with cell culture medium and cultured within an incubator with daily medium changes. For testing, glass slides were transferred and secured to the bottom plate of the rheometer and the top parallel plate was lowered to a gap height which ensured complete contact with the sample. A constant strain amplitude $(\gamma=0.01)$ frequency sweep $(\mathrm{f}=0.1-10 \mathrm{~Hz})$ was applied, with the measured elastic modulus ( $\left.G^{\prime}\right)$ serving as an indicator of overall cell-seeded construct stiffness. Moduli values measured at $0.1 \mathrm{~Hz}$ are reported in the text.

\section{Statistical analyses}

For each experiment, the sample size was $n=4$ and all experiments were repeated twice. Multi-factor ANOVA and post-hoc tests were used to determine the effects of scaffold type (NF, NF+MMP, or Matrigel), diabetic condition (db or wt), and culture time $(1,6$, or 14 days) on matrix remodeling (MMP-2, collagen I and IV expression), cell proliferation and apoptosis, and mechanical stiffness. All tests were run at a significance level of $\mathrm{p}=0.05$.

\section{Results}

Streptozotocin type I diabetes animal model results in substantial cardiac fibrosis at 6 weeks post STZ injection

Picrosirius red staining was performed on wild type and diabetic heart tissue sections. The staining demonstrated that mostly reticular, non-fibrillar collagen (appearing as green or white) was present in wild type control animal hearts (Figure 1A), while significant cardiac fibrosis was seen in the diabetic heart (Figure 1B; yellow, orange, and red staining) at 6 weeks post STZ injection. These results validate both the use of the STZ type I diabetic rat model as well as the 6 week time frame for cardiac fibrosis and diabetic cardiomyopathy development. Cardiac fibroblasts harvested from heart tissue of wild type and STZ type I diabetic rats were used for the following experiments.

\section{Peptide nanofibers provide a stable microenvironment for} controlled released of MMP-2

ELISA was used to measure the release kinetics of human MMP2 from RAD16-II nanofibers (Figure 2). Results showed a higher immediate release initially at $0.5 \mathrm{hr}(4.6 \pm 2.0 \%$ of total MMP- 2$), 1 \mathrm{hr}$ (2.5 $\pm 2.3 \%$ of total MMP-2) and $2 \mathrm{hrs}(1.1 \pm 0.5 \%$ of total MMP- 2$)$. After this initial burst, release slowed and remained relatively constant around $1 \%$ of total MMP-2 incorporated even as the time between supernatant collections increased. By $72 \mathrm{hrs}$, the total cumulative release was approximately $16 \%$ of the total MMP-2 incorporated initially into the nanofiber scaffold. This indicates successful MMP-2 incorporation into the NFs which allows for sustained protein release.

Peptide nanofibers provide a stable microenvironment, supporting long term cardiac fibroblast survival

Staining of wt and $\mathrm{db}$ cells both plated and embedded in
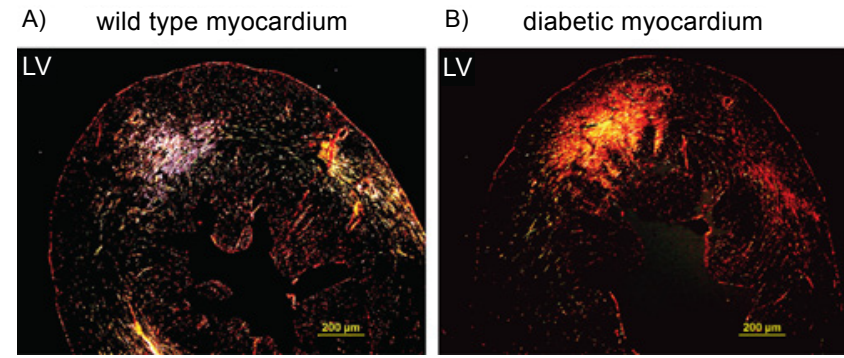

Figure 1: Evidence of cardiac fibrosis in the diabetic heart 6 weeks post streptozotocin injection. Picrosirius red staining of the rat myocardium shows the presence of cardiac fibrosis (golden red) in the left ventricle of heart tissue from STZ type I diabetic animals (right panel), as compared to heart tissue from age- and strain-matched wild type controls (left panel). Scale bar represents $200 \mu \mathrm{m}$ 
Citation: Hurley JR, Sheikh AQ, Huang W, Wang Y, Narmoneva DA, et al. (2012) Self Assembling Peptide Nanofibers for Extracellular Matrix Remodeling in Diabetic Cardiomyopathy. J Bioengineer \& Biomedical Sci S5:003. doi:10.4172/2155-9538.S5-003

MMP-2 Release from NFs

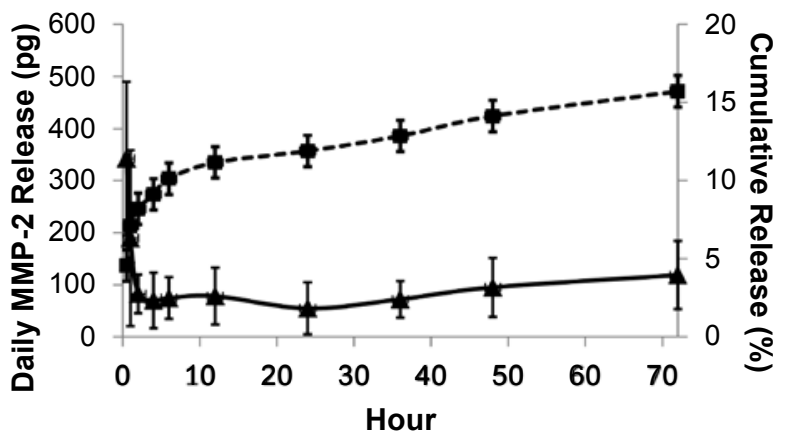

$\rightarrow$ Daily Release - - Cumulative Release

Figure 2: MMP-2 release kinetics from peptide nanofibers. Active human MMP-2 was incorporated into NFs at a concentration of $100 \mathrm{ng} / \mathrm{ml}$ and release kinetics were measure by performing ELISA on supernatant samples at $0.5,1$ $2,4,6,12,24,36,48$, and 72 hours. There was a higher immediate release initially at $0.5 \mathrm{hr}, 1$ and $2 \mathrm{hrs}$. After this initial burst, release slowed and remained relatively constant. By $72 \mathrm{hrs}$, the total cumulative release was approximately $16 \%$ of the total MMP-2 incorporated initially into the nanofiber scaffold.

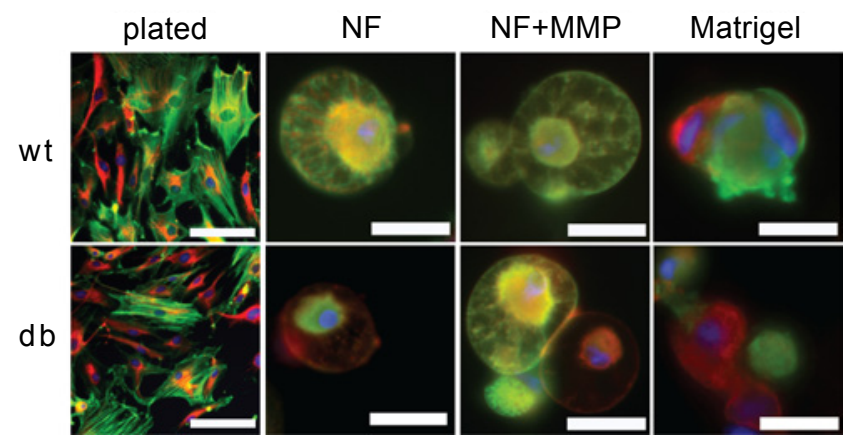

Figure 3: Staining for fibroblast phenotype. Cells were stained with antibodies for fibroblast marker vimentin (red) and myofibroblast marker $\alpha$-smooth muscle actin ( $\alpha$-SMA, green) and DAPI (blue). The top panels are wild type cells and the bottom panels are diabetic cells. Cells were either plated on gelatin coated dishes (left panels) or embedded in NF, NF+MMP, and Matrigel scaffolds (higher magnification panels, from left to right). All cells were positive for fibroblast marker vimentin and approximately $70-80 \%$ of cells were also positive for myofibroblast marker $\alpha$-SMA. Scale bar in left panels is $100 \mu \mathrm{m}$. Scale bar in higher magnification panels is $25 \mu \mathrm{m}$.

NF, NF+MMP and Matrigel was performed to confirm fibroblast phenotype and morphology within the scaffolds (Figure 3). All cells were positive for vimentin, an intermediate filament associated protein expressed by fibroblasts [35]. Additionally, approximately $70-80 \%$ of cells were also positive for $\alpha$-SMA, an intermediate filament associated protein expressed by myofibroblasts [36]. These observations are consistent with previous studies, where primary fibroblasts expressed a myofibroblast phenotype in culture, characterized by increased $\alpha$-SMA expression [38]. This activated fibroblast phenotype is critical in wound healing [36] and indicates an active matrix remodeling response by the fibroblasts in the nanofiber scaffold, which is important for the results of this study.

To determine if the peptide microenvironment supports longterm cardiac fibroblast survival and assess cell proliferation, LIVE/ $\mathrm{DEAD}^{\circ}$ and MTS-based cell proliferation assays were performed on cells embedded in NF, NF+MMP and Matrigel and cultured up to 14 days. Results from the LIVE/DEAD ${ }^{\circ}$ assay demonstrated that wild type and diabetic cell apoptosis levels (Figure 4) were less than $15 \%$ in NF, NF+MMP and Matrigel scaffolds. The number of apoptotic diabetic cells at day 1 was significantly higher than that in the wild type cells in Matrigel scaffolds $(p<0.05)$, with a similar trend observed between $\mathrm{db}$ and wt cells cultured in NF-based scaffolds. The results for later time points (days 6 and 14) demonstrate that this trend was actually reversed, with higher levels of apoptosis in the wt cells as compared to $\mathrm{db}$ cells ( $<<0.05$ for NF+MMP and Matrigel scaffolds). Interestingly, this increase in apoptosis at the later time points seemed to be compensated for by the increases in cell proliferation in the NFbased scaffolds, but not in Matrigel, with the total number of viable cells remaining at $95 \%$ or greater of the 125,000 total cells initially embedded within NF and NF+MMP scaffolds, with no significant differences observed between wild type and diabetic fibroblasts. Cell number (Figure 5) increased in both NF and NF+MMP scaffolds from day 1 to day 6 in both wt $(\mathrm{p}<0.05)$ and db cells $(\mathrm{p}<0.05$ for NF+MMP scaffold). No differences in numbers of either wild type or diabetic cells were observed with the addition of exogenous MMP-2 to the NFs.
A)

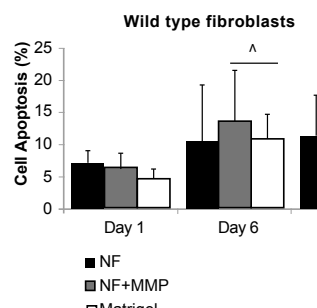

Matrigel
B)

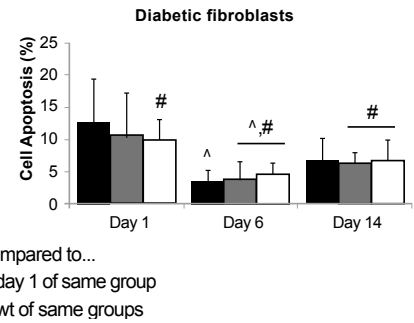

Figure 4: Cell apoptosis levels in cell-scaffold cultures. Cell apoptosis was measured using LIVE/DEAD $®$ staining. a) The percentage of apoptotic wild type (wt) fibroblasts was less than $15 \%$ for in NF, NF+MMP and Matrigel scaffolds at days 1, 6 and 14. While no difference was observed in apoptosis in NF scaffold, there was a significant increase in apoptosis in both NF+MMP and Matrigel from day 1 to days 6 and 14. b) The percentage of apoptotic diabetic (db) fibroblasts was less than $15 \%$ for in NF, NF+MMP and Matrigel scaffolds at days 1, 6 and 14. Initial (day 1) apoptosis levels in db fibroblasts were significantly higher and decreased at day 6 in all scaffolds. ${ }^{\wedge} p<0.05$ when compared to day 1 samples of same experimental group, $\# p<0.05$ when compared to wt samples of same experimental groups.

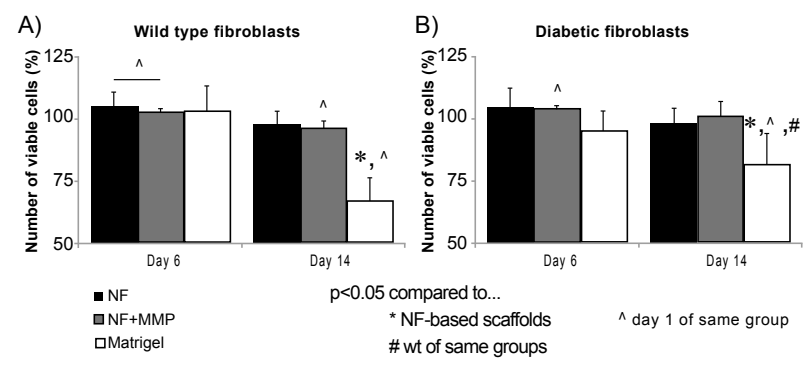

Figure 5: Cell viability levels in cell-scaffold cultures. Cell viability was measured using MTS-based proliferation assay at days 1, 6, and 14. a) The number of viable wild type (wt) cells in culture remained at $95 \%$ or greater of the 125,000 total cells initially embedded within NF and NF+MMP scaffolds. This was significantly higher than Matrigel cultures, where the number of viable cells by day 14 was less than $70 \%$ of initial cells numbers. b) The number of viable diabetic (db) cells in culture remained at $95 \%$ or greater of the 125,000 total cells initially embedded within NF and NF+MMP scaffolds. This was significantly higher than Matrigel cultures, where the number of viable cells by day 14 was at $82 \%$ of initial cells numbers. All data were normalized to day 1 values for analyses. * $p<0.05$ when compared to NF-based samples, ${ }^{\wedge} p<0.05$ when compared to day 1 samples of same experimental group, $\# p<0.05$ when compared to wt samples of same experimental groups. 
In contrast, in Matrigel cultures, the number of viable cells by day 14 was significantly decreased in both wild type and diabetic fibroblasts $(\mathrm{p}<0.05)$, with the cell number significantly lower than in NF and $\mathrm{NF}+\mathrm{MMP}$ scaffolds $(\mathrm{p}<0.05)$.

Overall, these results indicate a balance between cell proliferation and apoptosis in the NF and NF+MMP scaffolds, in contrast to the shift toward more apoptosis with time in culture in Matrigel. Therefore, these results suggest an overall stability of the peptide nanofiber microenvironment both with and without added MMP-2, which supports long term cell survival in both wild type and diabetic fibroblasts in vitro.

\section{Expression of native MMP-2 by cardiac fibroblasts is increased in peptide nanofiber microenvironment}

Native rat MMP-2 concentration expressed by cardiac fibroblasts was measured in both the media and matrix using a rat MMP-2 ELISA (Figure 6). In media samples (Figures 6A-B), there was no difference in MMP-2 expression levels by wt cells between different time points in NF-based scaffolds. However, MMP-2 expression by db cells in these scaffolds was lower at day 1 ( $p<0.05$ vs. wt NF and NF+MMP) and subsequently increased in the NF group ( $p<0.05$ vs. day 1 ). No differences in native MMP-2 media expression by wild type cells was observed as a result of the addition of exogenous MMP-2 to the NFs,
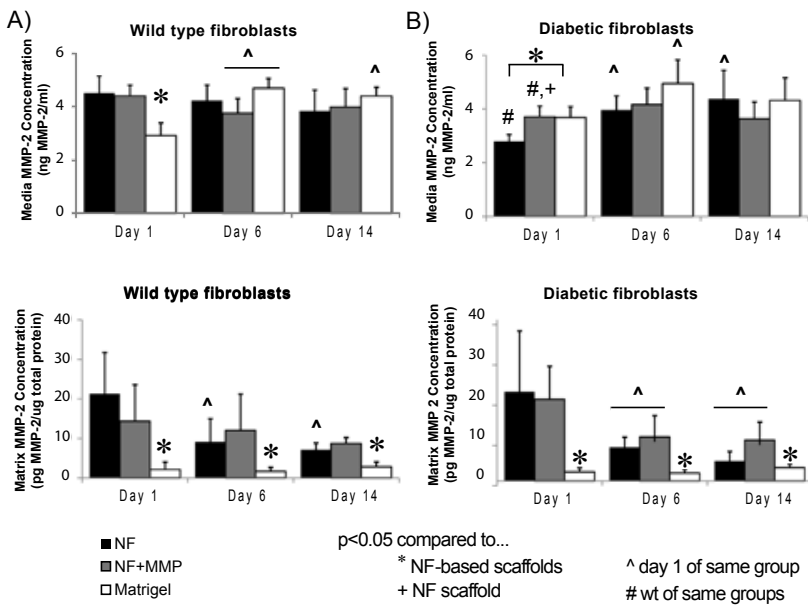

Figure 6: Native protein expression of matrix metalloproteinase -2. ELISA was performed on samples collected from cell-scaffold constructs at days 1 , 6 and 14 to determine concentration of native rat MMP-2 in the media (panels a and b) and matrix-bound (panels $c$ and d). a) Native MMP-2 protein expression (ng MMP-2/ml) by wild type (wt) fibroblasts in NF, NF+MMP and Matrigel scaffolds as measured in media samples. MMP-2 expression was increased at day 1 in NF as compared to Matrigel, with no effect of exogenous MMP-2 (NF+MMP). b) Native MMP-2 protein expression (ng MMP-2/ml) by diabetic (db) fibroblasts in NF, NF+MMP and Matrigel scaffolds as measured in media samples. A temporal increase in MMP-2 expression was observed in NF scaffolds. c) Native MMP-2 protein expression (pg MMP-2/ug total protein) by wild type (wt) fibroblasts in NF, NF+MMP and Matrigel scaffolds as measured in matrix samples. MMP2 expression was increased in NF-based scaffolds as compared to Matrigel, with no effect of exogenous MMP-2 (NF+MMP). A temporal decrease was observed in MMP-2 expression in NF scaffolds. d) Native MMP-2 protein expression (pg MMP-2/ug total protein) by diabetic (db) fibroblasts in NF, NF+MMP and Matrigel scaffolds as measured in matrix samples. MMP-2 expression was again increased in NF-based scaffolds as compared to Matrigel, with no effect of exogenous MMP-2 (NF+MMP). A temporal decrease was again observed in MMP-2 expression in NF scaffolds. * $p<0.05$ when compared to NF-based samples, $+p<0.05$ when compared to NF samples, ${ }^{\wedge} p<0.05$ when compared to day 1 samples of same experimental group, \# $p<0.05$ when compared to wt samples of same experimental groups.
A)

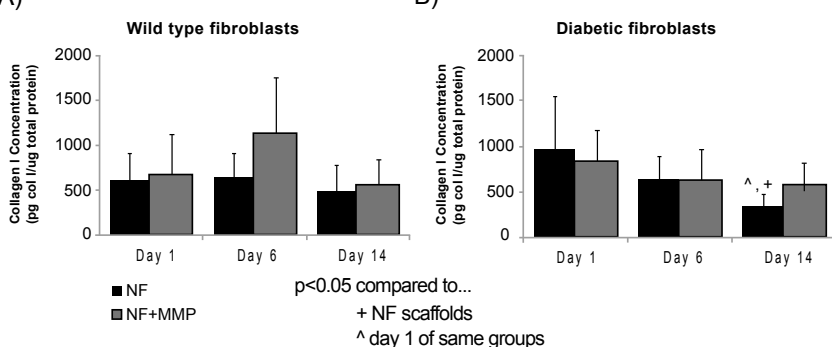

Figure 7: Collagen I deposition. ELISA was performed on cell-scaffold matrix samples for wild type fibroblasts (panel a) and diabetic fibroblasts (panel b) at days 1,6 , and 14 to determine protein levels of collagen I (col I). a) Collagen I concentration (pg col I/ug total protein) for wild type (wt) fibroblasts in NF and NF+MMP scaffolds at days 1,6 and 14. Steady collagen I levels were observed in NF, with no significant effect of exogenous MMP- 2 (NF+MMP). b) Collagen I concentration (pg col l/ug total protein) for diabetic (db) fibroblasts in NF and $\mathrm{NF}+\mathrm{MMP}$ scaffolds at days 1,6 and 14. A significant decrease in collagen I levels was observed in NF by day 14 consistent with ECM remodeling. The addition of exogenous MMP-2 (NF+MMP) resulted in significantly higher collagen I levels at day 14 as compared to NF. Expression levels were normalized using total protein content as measured using Bradford assay. $+p<0.05$ when compared to NF samples, ${ }^{\wedge} \mathrm{p}<0.05$ when compared to day 1 samples of same experimental group.

although in diabetic cells a significant increase at day 1 was observed between NF and NF+MMP scaffolds $(\mathrm{p}<0.05)$. In contrast to the MMP2 levels observed in NF-based samples, MMP-2 levels in Matrigel samples were lowest at day 1 in both $\mathrm{db}$ and wt cells and increased with time ( $\mathrm{p}<0.05$ vs. day 1$)$. Additionally, MMP-2 expression by wt cells in Matrigel scaffolds at day 1 was significantly less than NFbased scaffolds $(\mathrm{p}<0.05)$. The amount of MMP-2 bound to the NF and $\mathrm{NF}+\mathrm{MMP}$ matrix (Figure 6C-D) showed an opposite trend from that in the media, with the highest MMP-2 concentration at day 1 , which significantly decreased by day 6 in both wild type and diabetic fibroblasts $(\mathrm{p}<0.05)$. Additionally, matrix-bound MMP-2 levels by both wt and $\mathrm{db}$ fibroblasts were significantly higher in both NF and NF+MMP scaffolds than in Matrigel at each time point $(\mathrm{p}<0.05)$. No differences in native MMP-2 levels in the matrix by wild type or diabetic fibroblasts were observed as a result of the addition of exogenous MMP-2 to the NFs.

\section{Native extracellular matrix deposition by cardiac fibroblasts} is supported by peptide nanofiber microenvironment

Collagen I (col I) and Collagen IV (col IV) ELISAs on matrix samples were performed to determine the concentration of native ECM deposited by wild type and diabetic fibroblasts. Due to the fact that the Matrigel scaffold itself contains a significant amount of col I and IV, no comparison with NF and NF+MMP groups were made.

There were no differences seen in collagen I deposition by wild type cells at any time point in NF and NF+MMP scaffolds (Figure 7). Additionally, no significant differences in collagen I deposition by wild type fibroblasts were observed as a result of the addition of exogenous MMP-2 to the NFs. However, in diabetic cells, there was a significant temporal decrease in collagen I levels in NF scaffolds $(\mathrm{p}<0.05)$, where collagen I levels were significantly lower at day 14 as compared to $\mathrm{NF}+\mathrm{MMP}$ scaffolds $(\mathrm{p}<0.05)$.

Interestingly, both wt and $\mathrm{db}$ cells demonstrated a temporal decrease in collagen IV levels (Figure 8), similar to that observed in collagen I levels in diabetic cells only. This trend was significant in wild type cells, where deposition of col IV was significantly higher in NF- 
A)

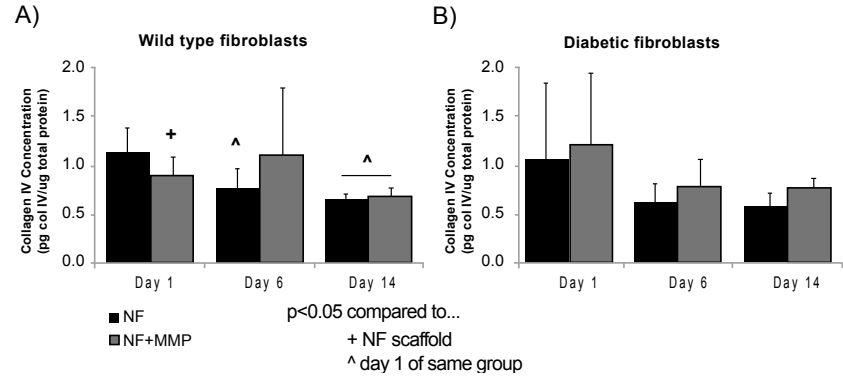

Figure 8: Collagen IV deposition. ELISA was performed on cell-scaffold matrix samples for wild type fibroblasts (panel a) and diabetic fibroblasts (panel b) at days 1,6 , and 14 to determine protein levels of collagen IV (col IV). a) Collagen IV concentration (pg col IV/ug total protein) for wild type (wt) fibroblasts in NF and NF+MMP scaffolds at days 1,6 and 14. Collagen IV levels at day 1 were higher in NF than in NF+MMP scaffolds and decreased with time, indicating ECM remodeling. b) Collagen IV concentration (pg col IV/ug total protein) for diabetic (db) fibroblasts in NF and NF+MMP scaffolds at days 1,6 and 14. Collagen IV levels were highest at day 1 in NF scaffolds, with no effect of exogenous MMP-2 (NF+MMP). Expression levels were normalized using total protein content as measured using Bradford assay. $+p<0.05$ when compared to NF samples, ${ }^{\wedge} \mathrm{p}<0.05$ when compared to day 1 samples of same experimental group.
A)

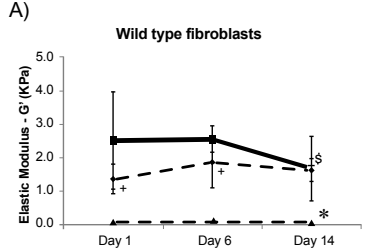

B)

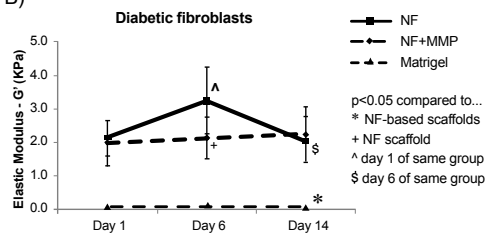

Figure 9: Stiffness of cell-scaffold constructs measured using rheometry. a) Elastic moduli (G') values in $\mathrm{kPa}$ for wild type (wt) fibroblasts in NF, NF+MMP and Matrigel scaffolds at days 1,6 and 14. NF and NF+MMP scaffolds are significantly stiffer than Matrigel at all time points. Decreased stiffness was observed in NF scaffolds at day 14. No changes in stiffness were observed in $\mathrm{NF}+\mathrm{MMP}$ scaffolds. b) Elastic moduli ( $\mathrm{G}^{\prime}$ ) values in $\mathrm{kPa}$ for diabetic (db) fibroblasts in NF, NF+MMP and Matrigel scaffolds at days 1,6 and 14. Again, NF and NF+MMP scaffolds are significantly stiffer than Matrigel at all time points. NF scaffolds exhibited a significant increase in stiffness at day 6 followed by a significant decrease at day 14 . No changes in stiffness were observed in NF+MMP scaffolds. G' $(\mathrm{kPa})$ is reported at frequency of $0.1 \mathrm{~Hz}$. ${ }^{*} \mathrm{p}<0.05$ when compared to NF-based samples, $+p<0.05$ when compared to NF samples, $A$ $p<0.05$ when compared to day 1 samples of same experimental group, $\$ p<0.05$ when compared to day 6 samples of same experimental group.

based scaffolds initially at day 1 than later time points $(\mathrm{p}<0.05)$. There was significantly more collagen IV deposited by wild type cells at day 1 in NF than in NF+MMP scaffolds $(\mathrm{p}<0.05)$.

\section{The extracellular mechanical environment is regulated by matrix remodeling by cardiac fibroblasts}

A parallel-plate rheometer was used to measure the mechanical properties of cell-scaffold constructs at days 1, 6, and 14, with elastic modulus (G') serving as an indicator of construct stiffness (Figure 9). Previous studies indicate that overall cell-scaffold stiffness is regulated by a combination of extracellular matrix deposition, remodeling and scaffold disruption due to cell migration [26]. Scaffold-only controls at day 1 served as a baseline of scaffold stiffness (NF: $1.93 \pm 0.78 \mathrm{kPa}$, NF+MMP: $1.38 \pm 0.21 \mathrm{kPa}$, Matrigel: $0.09 \pm 0.03 \mathrm{kPa})$, with NF-based scaffolds significantly stiffer than Matrigel $(\mathrm{p}<0.05)$. For wt cells, no difference in NF scaffold stiffness was observed from day 1 to day 6 . However, day 14 stiffness was significantly decreased from day 6 in
NF scaffolds $(p<0.05)$. In $d b$ cells, there was a significant increase in stiffness from day 1 to $6(\mathrm{p}<0.05)$, and again a significant decrease from day 6 to day $14(\mathrm{p}<0.05)$. In NF+MMP scaffolds, there was no difference in scaffold stiffness observed with time in either wild type or diabetic cell constructs. At all time points for both wild type and diabetic fibroblasts, both NF and NF+MMP scaffolds were significantly stiffer than Matrigel scaffolds $(\mathrm{p}<0.05)$, indicating the robust structural integrity of the peptide nanofibers.

\section{Discussion}

The results of this study demonstrate that nanofiber microenvironment supports slow MMP-2 release without detrimental effects of increased MMP-2 levels (both native and exogenous) on cardiac fibroblast viability and behavior, indicating the promise of this material for therapies which may involve MMP-mediated tissue remodeling. Interestingly, there was no apparent effect of the presence of exogenous MMP in the NF scaffold on extracellular matrix remodeling, with both of the NF-based scaffolds stimulating significantly improved matrix remodeling response by cardiac fibroblasts as compared to Matrigel controls. Overall, the results demonstrate long-term survival and temporal in vitro matrix remodeling by both wild type and diabetic cardiac fibroblasts in the NFs (Figure 10). Early at day 1, we see increased native MMP-2 expression and ECM deposition. By day 6, MMP-2 expression is still prominent with stiffness values consistent with ECM levels. However, by day 14 we see a shift towards more active matrix remodeling, with decreased MMP-2 and ECM levels and decreased stiffness. These results are consistent with the normal wound healing response in cardiac tissue in vivo, which is characterized by initial increased MMP expression leading to net ECM degradation [39].

RAD16-II nanofibers were investigated in this study as a biomaterial strategy to increase local MMP-2 concentration, either via stimulation of native expression or delivery of exogenous protein, in order to promote extracellular matrix remodeling by cardiac fibroblasts in vitro. An RAD16-II peptide nanofibers approach was chosen based on ease of handling, proteolytic stability, and proven myocardial protein delivery capability $[21,22,24]$. Importantly, RAD16-II peptide nanofibers have been shown to significantly enhance native expression of MMP-2 by human dermal fibroblasts as compared with collagen I controls [26], which is particularly interesting as collagen I has been identified as a stimulus for MMP-2 activation [33, 40]. The results from this study extend this prior observation to another control scaffold,

\section{NFs Support Temporal Matrix Remodeling by Cardiac Fibroblasts}

\begin{tabular}{|c|c|c|}
\hline $\begin{array}{l}\text { MMP Expression } \\
\text { and ECM Deposition }\end{array}$ & & $\begin{array}{l}\text { Active Matrix } \\
\text { Remodeling }\end{array}$ \\
\hline 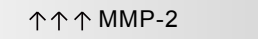 & 个个MMP-2 & 个 MMP-2 \\
\hline 个Col I & $\uparrow$ Col I & $\downarrow \mathrm{Col} ।$ \\
\hline$\uparrow$ Col IV & $\downarrow$ Col IV & $\downarrow$ Col IV \\
\hline$\uparrow$ stiffness & $\uparrow$ stiffness & $\downarrow$ stiffness \\
\hline
\end{tabular}

Figure 10: Proposed schematic of extracellular matrix remodeling response by cardiac fibroblasts (both wild type and diabetic) in the peptide nanofiber scaffold. Increased native MMP-2 expression and ECM deposition by cardiac fibroblasts is seen early at day 1. By day 6, MMP-2 expression is still prominent with stiffness values consistent with observed ECM levels. However, a shift towards more active matrix remodeling is seen by day 14 , with decreased MMP-2 and ECM levels and decreased stiffness. 
with the RAD16- II nanofibers stimulating significantly higher native MMP-2 expression by cardiac fibroblasts, as compared to Matrigel controls. Furthermore, the measurement of cell-scaffold stiffness indicates that NF scaffolds retained their structural integrity and stability in the presence of high MMP-2 levels, likely due to absence of proteolytic degradation sites in the NF sequence. Importantly, our results also demonstrate that the NF microenvironment is supportive for diabetic, as well as normal, cardiac fibroblasts, in contrast to significantly increased death for both cell types in Matrigel controls at day 14 in culture. In fact, we have observed that the peptide nanofiber microenvironment consistently supports increased levels of MMP2 accompanied by low levels of apoptosis [26]. Previous studies in experimental models of diabetic cardiomyopathy have demonstrated decreased expression and activity of MMP-2 concomitant with increased cardiac apoptosis [9]. Further, it has been shown that MMP-2-mediated chemokine cleavage plays an important role in myocardial protection against inflammation-associated damage [41]. Additionally, in vitro studies on human umbilical vein endothelial cells have demonstrated increased MMP-2 activity results in a reduction in high glucose-induced cell apoptosis [42]. Such studies along with our results may indicate a potential connection between increased MMP- 2 levels and increased cell survival in diabetic conditions [42,43]. Overall, our data indicates a balance between cell proliferation and apoptosis and an overall stability in the peptide nanofiber microenvironment which supports long term cell survival in cardiac fibroblasts in vitro, consistent with the previous studies of long term culture of endothelial cells in this microenvironment [26].

MMPs are regulated in vivo by inhibition via endogenous tissue inhibitors of metalloproteinases (TIMPs), with TIMP-1 and -2 predominantly produced by cardiac fibroblasts [40]. In addition to its role as an endogenous inhibitor, TIMP- 2 can form a complex with membrane-tethered membrane type 1 (MT1)-MMP and pro-MMP-2, leading to activation of MMP-2 [44] and therefore plays an important role in both inhibition and activation of MMP-2. In animal models of diabetes and diabetic cardiomyopathy, studies have demonstrated increased levels of TIMP-2 gene expression and protein levels $[14,45]$ and MT1-MMP expression [46] concomitant with reduced expression and activity of MMP-2 $[9,14-16,46]$. Additionally, in a study of human patients with diabetes, increased levels of circulating TIMP-1 have been observed [5]. These previous studies may provide a mechanistic understanding to the observed cardiac fibrosis in diabetes, as not only is expression of both MMP-2 and its activator MT1- MMP decreased in diabetes, but TIMP levels are increased, contributing to diminished remodeling of the extracellular matrix. Our previous studies have demonstrated that the peptide nanofiber microenvironment supports increased levels of MMP-2/TIMP-2 complexes in vitro [26] which may indicate increased MMP-2 activation in our system. Therefore, the improved extracellular matrix degradation and remodeling observed in this study may be the result of increased MMP-2 activation due to increased MMP-2/TIMP-2 complexes in the nanofiber microenvironment. Future studies will be directed toward comprehensive analysis of the mechanism of MMP-2 activation in the nanofiber microenvironment and the roles of TIMPs in this process.

While our results demonstrate that NFs alone do increase native MMP-2 expression, we were also interested in the use of NFs as an exogenous protein delivery system. An RAD16-II scaffold-based approach for protein delivery is particularly attractive, because it allows for temporally-controlled and localized delivery, and can be achieved via either diffusion from or tethering to a scaffold, depending on protein size and binding affinity [47]. Additionally, increased peptide nanofiber density results in decreased protein diffusion [25], suggesting a straight-forward strategy for controlling protein release kinetics. Recent in vitro studies have examined the release of functional proteins and cytokines, from similar nanofiber scaffolds and have shown slow and sustained release profiles over 2 to 3 weeks, with diffusion through nanofibers dependent primarily on protein size $[25,48]$. For this study, we investigated the release kinetics of human MMP-2 from the RAD16-II nanofibers. The data showed that NF scaffolds can be effectively used as a MMP-2 delivery vehicle, with a faster initial release burst slowing to a more steady release rate and reaching a cumulative release of $16 \%$ by day 3 . These results are consistent with previous studies on protein release from hydrogel scaffolds [25], with prolonged in vitro release of the incorporated MMP-2 due to entrapment in the highly entangled nanofiber structure. However, after delivery to the myocardium the peptide nanofibers would be subjected to intensive cell migration, particularly by highly invasive cardiac fibroblasts, leading to structural deformation and disruption of the nanofiber network [49] and increased release of the incorporated protein into the surrounding tissue. Thus, previous studies have shown temporal decrease (up to 28 days) in retention of IGF-1 incorporated into NFs and injected into the myocardium of rats [21]. Additionally, studies have shown that RAD16-II remained present 28 days after treatment in diabetic wounds [50] and after injection into the myocardium in mice and rats $[21,51]$ without significant immune response, allowing for slow and sustained protein release.

Interestingly however, while the RAD16-II nanofibers clearly are effective as a protein delivery vehicle, no clear significant increase in fibroblast matrix remodeling response was seen with incorporation of exogenous MMP-2 into NFs. Therefore, the results from this study suggest that for cardiac tissue remodeling, using the NFs alone to stimulate native MMP-2 expression may ultimately be the more suitable strategy due to a better risk-benefit value by removing the need to add further complexity to cardiac tissue engineering strategies through the addition of an exogenous agent such as MMP-2 [52]. This strategy may potentially be applied in vivo using the DCM model and injecting NFs into the fibrotic left ventricular wall of the myocardium as a regenerative stimulus. Nanofibers have been previously injected into the myocardium at multiple sites either alone [51] or as a delivery vehicle for exogenous protein and/or cells $[22,24,53,54]$ with promising results. By injecting nanofibers at multiple sites in the fibrotic diabetic heart muscle, the potential stimulatory effects will be pervasive throughout the tissue, attenuating the observed MMP-2 deficiency and inducing reparative matrix remodeling and cardiac regeneration.

From a clinical perspective, diabetes leads to high myocardial stiffness which is ultimately associated with a poorer prognosis and heart failure for patients [55-57]. Cardiac cells, including cardiac fibroblasts and cardiomyocytes, play an important role in the development increased stiffness in the diabetic heart $[18,43,58]$, which may result from excessive fibrosis, high cardiomyocyte stiffness and deposition of advanced glycation end products (AGEs) [59-61]. This study focused on the fibrotic mechanism for diabetic cardiomyopathy and a potential strategy to address it using the nanofiber technology. In this respect, previous studies suggest the major role for cardiac fibroblasts in both physiological and pathological extracellular matrix homoeostasis [40,62-64], as well as dysregulation of physiological cardiac matrix remodeling under diabetic conditions $[14,15,18]$. These observations suggest that diabetic conditions may lead to an impaired matrix remodeling response by cardiac fibroblasts via a MMP-related mechanism. Therefore, cardiac fibroblasts were the primary cell type investigated in our study. The results of this study suggest that 
Citation: Hurley JR, Sheikh AQ, Huang W, Wang Y, Narmoneva DA, et al. (2012) Self Assembling Peptide Nanofibers for Extracellular Matrix Remodeling in Diabetic Cardiomyopathy. J Bioengineer \& Biomedical Sci S5:003. doi:10.4172/2155-9538.S5-003

Page 8 of 10

this impairment in matrix remodeling may be attenuated through stimulation of native MMP-2 expression in the RAD16-II peptide nanofiber scaffold, as relatively small differences in matrix remodeling response were seen between diabetic and wild type cardiac fibroblasts. Additionally, previous studies have demonstrated that cardiac fibroblasts also respond to pro-fibrotic cytokines (i.e. TGF- $\beta$ ) which are expressed by cardiomyocytes $[43,64]$ in high glucose conditions. In addition to causing cardiac fibroblast activation and stimulating fibrotic remodeling, TGF- $\beta 1$ can contribute to cardiac dysregulation via an autocrine loop involving SMAD signaling $[43,65]$. Therefore, investigating the intercellular signaling between cardiomyocytes and cardiac fibroblasts is extremely important for understanding the overall mechanisms of cardiac remodeling in diabetes. Our previous studies [26] elucidated the chemical and mechanical regulation of capillary morphogenesis by fibroblasts and demonstrated that the nanofiber microenvironment supports endothelial-fibroblasts angiogenic interactions in vitro. Our results suggest that the nanofiber microenvironment can promote matrix remodeling without excessive stiffening (which may be caused by cell-mediated contraction seen in other scaffolds $[66,67])$. Therefore, the nanofiber technology may provide an attractive platform, as it allows for studies of cell-cell interactions and paracrine signaling using three-dimensional coculture of cardiac fibroblasts, cardiomyocytes and/or endothelial cells in the chemically-controlled pro-angiogenic microenvironment $[26,68]$, emphasizing the potential of this material for cardiac tissue engineering applications.

In summary, the findings from our study may contribute the development of novel therapeutic strategies for the promotion of reparative matrix remodeling and cardiac regeneration in diabetic cardiomyopathy. DCM leads to excess collagen deposition, myocardial fibrosis, and cardiac hypertrophy [2,5]. The prevention and treatment of DCM is a clinically relevant and active research focus, with studies suggesting that glycemic control is beneficial early in myocardial dysfunction [3], however late diagnoses of diabetes and/or DCM may limit this preventative measure. Neurohormonal antagonism has demonstrated preserved diastolic function in the diabetic heart in animal models $[3,7]$, however, it has not yet been translated to clinical studies. While strategies such as these appear promising, there still exists a need for novel approaches for treatment of diabetic cardiomyopathy which may focus on alternative molecular mechanisms for the disease, including cardiac dysregulation of MMP-2 expression and activity $[9,14-$ 16]. In this study, we observed that increased MMP-2 concentration resulting from the nanofiber microenvironment improved in vitro matrix remodeling by cardiac fibroblasts, both wild type and diabetic. Furthermore, RAD16-II and similar nanofibers are easily administered and have been locally injected into the myocardium either alone [51] or as a protein delivery vehicle $[21,22,24]$. Therefore, the results of this study suggest that peptide nanofibers may be a uniquely suited cardiac tissue engineering substrate to increase local MMP-2 concentration in the diabetic heart, leading to therapeutic matrix remodeling and cardiac regeneration.

\section{Acknowledgments}

This project was supported by the American Heart Association (AHA BGIA 0765425B, DAN \& GRA 09PRE2150073, JRH), the National Institutes of Health (NIH 1R21DK078814- 01A1, DAN), the National Science Foundation (NSFIGERT 0333377, JRH), and the University of Cincinnati Department of Biomedical Engineering (DAN). The authors thank Meredith Beckenhaupt, Cameron Ingram, and Andrew Mutchler for their experimental assistance.

\section{References}

1. ADA (2010) American Diabetes Association.

2. Asbun J, Villarreal FJ (2006) The pathogenesis of myocardial fibrosis in the setting of diabetic cardiomyopathy. J Am Coll Cardiol 47: 693-700.

3. Aneja A, Tang WH, Bansilal S, Garcia MJ, Farkouh ME (2008) Diabetic Cardiomyopathy: Insights into Pathogenesis, Diagnostic Challenges, and Therapeutic Options. American Journal of Medicine 121: 748-757.

4. Boudina S, Abel ED (2007) Diabetic cardiomyopathy revisited. Circulation 115 3213-3223.

5. Tayebjee MH, Lip GY, MacFadyen RJ (2005) What role do extracellular matrix changes contribute to the cardiovascular disease burden of diabetes mellitus? Diabet Med 22: 1628-1635.

6. Spinale FG (2007) Myocardial matrix remodeling and the matrix metalloproteinases: Influence on cardiac form and function. Physiol Rev 87 1285-1342.

7. Hayashi T, Sohmiya K, Ukimura A, Endoh S, Mori T, et al. (2003) Angiotensin II receptor blockade prevents microangiopathy and preserves diastolic function in the diabetic rat heart. Heart 89: 1236-1242.

8. Lorenzo O, Picatoste B, Ares-Carrasco S, Ramírez E, Egido J, et al. (2011) Potential role of nuclear factor $\mathrm{kb}$ in diabetic cardiomyopathy. Mediators Inflamm 2011: 652097

9. Westermann D, Rutschow S, Jäger S, Linderer A, Anker S, et al. (2007) Contributions of inflammation and cardiac matrix metalloproteinase activity to cardiac failure in diabetic cardiomyopathy: The role of angiotensin type 1 receptor antagonism. Diabetes 56: 641-646.

10. Tyagi SC, Hayden MR (2003) Role of nitric oxide in matrix remodeling in diabetes and heart failure. Heart Fail Rev 8: 23-28.

11. Tschöpe C, Walther T, Königer J, Spillmann F, Westermann D, et al. (2004) Prevention of cardiac fibrosis and left ventricular dysfunction in diabetic cardiomyopathy in rats by transgenic expression of the human tissue kallikrein gene. FASEB Journal 18: 828-835.

12. Portik-Dobos V, Anstadt MP, Hutchinson J, Bannan M, Ergul A, et al. (2002) Evidence for a matrix metalloproteinase induction/activation system in arterial vasculature and decreased synthesis and activity in diabetes. Diabetes 51 : 3063-3068.

13. Lewandowski KC (2011) Matrix metalloproteinases in type 2 diabetes and nondiabetic controls: Effects of short-term and chronic hyperglycaemia. Archives of Medical Science 7: 294-303.

14. Van Linthout S, Seeland U, Riad A, Eckhardt O, Hohl M, et al. (2008) Reduced MMP-2 activity contributes to cardiac fibrosis in experimental diabetic cardiomyopathy. Basic Res Cardiol 103: 319-327.

15. Bollano E, Omerovic E, Svensson H, Waagstein F, Fu M (2007) Cardiac remodeling rather than disturbed myocardial energy metabolism is associated with cardiac dysfunction in diabetic rats. International Journal of Cardiology 114: 195-201.

16. Zhang N, Li J, Luo R, Jiang J, Wang JA (2008) Bone marrow mesenchymal stem cells induce angiogenesis and attenuate the remodeling of diabetic cardiomyopathy. Exp Clin Endocrinol Diabetes 116: 104-111.

17. Tokudome T, Horio T, Yoshihara F, Suga S, Kawano Y, et al. (2004) Direct effects of high glucose and insulin on protein synthesis in cultured cardiac myocytes and DNA and collagen synthesis in cardiac fibroblasts. Metabolism 53: 710-715

18. Asbun J, Manso AM, Villarreal FJ (2005) Profibrotic influence of high glucose concentration on cardiac fibroblast functions: Effects of losartan and vitamin $\mathrm{E}$. Am J Physiol Heart Circ Physiol 288: H227- H234.

19. Amiri F, Shaw S, Wang X, Tang J, Waller JL, et al. (2002) Angiotensin II activation of the JAK/STAT pathway in mesangial cells is altered by high glucose. Kidney Int 61: 1605-1616.

20. Lerman OZ, Galiano RD, Armour M, Levine JP, Gurtner GC (2003) Cellula dysfunction in the diabetic fibroblast: Impairment in migration, vascular endothelial growth factor production, and response to hypoxia. Am J Patho 162: 303-312.

21. Davis ME, Hsieh PC, Takahashi T, Song Q, Zhang S, et al. (2006) Loca myocardial insulin-like growth factor 1 (IGF-1) delivery with biotinylated peptide 
Citation: Hurley JR, Sheikh AQ, Huang W, Wang Y, Narmoneva DA, et al. (2012) Self Assembling Peptide Nanofibers for Extracellular Matrix Remodeling in Diabetic Cardiomyopathy. J Bioengineer \& Biomedical Sci S5:003. doi:10.4172/2155-9538.S5-003

nanofibers improves cell therapy for myocardial infarction. Proc Natl Acad Sci USA 103: 8155-8160

22. Segers VF, Tokunou T, Higgins LJ, MacGillivray C, Gannon J, et al. (2007) Local delivery of protease-resistant stromal cell derived factor- 1 for stem cell recruitment after myocardial infarction. Circulation 116: 1683-1692.

23. Zhang S (2003) Fabrication of novel biomaterials through molecular selfassembly. Nat Biotechnol 21: 1171-1178.

24. Hsieh PC, Davis ME, Gannon J, MacGillivray C, Lee RT (2006) Controlled delivery of PDGF-BB for myocardial protection using injectable self-assembling peptide nanofibers. J Clin Invest 116: 237-248.

25. Koutsopoulos S, Unsworth LD, Nagai Y, Zhang S (2009) Controlled release of functional proteins through designer self-assembling peptide nanofiber hydrogel scaffold. Proc Natl Acad Sci U S A 106: 4623-4628.

26. Hurley JR, Balaji S, Narmoneva DA (2010) Complex temporal regulation of capillary morphogenesis by fibroblasts. Am J Physiol Cell Physiol 299: C444-C453.

27. Cheung PY (1999) Inhibition of matrix metalloproteinase-2 (MMP-2) improves recovery of mechanical function following ischemia-reperfusion in isolated rat hearts. Proceedings of the Western Pharmacology Society 42: 132.

28. Lombardi R, Betocchi S, Losi MA, Tocchetti CG, Aversa M, et al. (2003) Myocardial collagen turnover in hypertrophic cardiomyopathy. Circulation 108: 1455-1460.

29. Bugger H, Abel ED (2009) Rodent models of diabetic cardiomyopathy. Dis Model Mech 2: 454-466.

30. Mihm MJ, Seifert JL, Coyle CM, Bauer JA (2001) Diabetes related cardiomyopathy time dependent echocardiographic evaluation in an experimental rat model. Life Sci 69: 527-542

31. Coleman, R (2011) Picrosirius red staining revisited. Acta Histochemica 113 231-233.

32. Narmoneva DA, Oni O, Sieminski AL, Zhang S, Gertler JP, et al. (2005) Self-assembling short oligopeptides and the promotion of angiogenesis. Biomaterials 26: 4837-4846.

33. Thompson EW, Yu M, Bueno J, Jin L, Maiti SN, et al. (1994) Collagen induced MMP-2 activation in human breast cancer. Breast Cancer Res Treat 31: 357 370

34. Coker ML, Doscher MA, Thomas CV, Galis ZS, Spinale FG (1999) Matrix metalloproteinase synthesis and expression in isolated LV myocyte preparations. Am J Physiol 277: H777-H787.

35. Strutz F, Okada H, Lo CW, Danoff T, Carone RL, et al. (1995) Identification and characterization of a fibroblast marker: FSP1. J Cell Biol 130: 393-405.

36. Darby, I., O. Skalli, and G. Gabbiani (1990) Alpha-Smooth muscle actin is transiently expressed by myofibroblasts during experimental wound healing. Lab Invest 63: 21-29.

37. Myers PR, Tanner MA (1998) Vascular endothelial cell regulation of extracellular matrix collagen: Role of nitric oxide. Arterioscler Thromb Vasc Biol 18: 717-722.

38. Mughal RS, Warburton P, O'Regan DJ, Ball SG, Turner NA, et al. (2009) Peroxisome proliferator-activated receptor $y$-independent effects of thiazolidinediones on human cardiac myofibroblast function. Clin Exp Pharmacol Physiol 36: 478-486.

39. Brown RD, Ambler SK, Mitchell MD, Long CS (2005) The cardiac fibroblast: therapeutic target in myocardial remodeling and failure. Annu Rev Pharmaco Toxicol 45: 657-87

40. Morley ME, Riches K, Peers C, Porter KE (2007) Hypoxic inhibition of human cardiac fibroblast invasion and MMP-2 activation may impair adaptive myocardial remodelling. Biochem Soc Trans 35: 905-907.

41. Westermann D, Savvatis K, Lindner D, Zietsch C, Becher PM (2011) Reduced Degradation of the Chemokine MCP-3 by Matrix Metalloproteinase-2 Exacerbates Myocardial Inflammation in Experimental Viral Cardiomyopathy. Circulation 124: 2082-2093

42. Ho FM, Liu SH, Lin WW, Liau CS (2007) Opposite effects of high glucose on MMP-2 and TIMP-2 in human endothelial cells. J Cell Biochem 101: 442-450.

43. Ares-Carrasco S, Picatoste B, Benito-Martín A, Zubiri I, Sanz AB, et al. (2009)
Myocardial fibrosis and apoptosis, but not inflammation, are present in long-term experimental diabetes. Am J Physiol Heart Circ Physiol 297: H2109-H2119.

44. Bernardo MM, Fridman R (2003) TIMP-2 (tissue inhibitor of metalloproteinase-2 regulates MMP-2 (matrix metalloproteinase-2) activity in the extracellula environment after pro-MMP-2 activation by MT1 (membrane type 1)-MMP. Biochem J 374: 739-745

45. Li Q, Sun SZ, Wang Y, Tian YJ, Liu MH (2007) The roles of MMP-2/TIMP-2 in extracellular matrix remodelling in the hearts of STZ-induced diabetic rats. Acta Cardiol 62: 485-491.

46. Jesmin S, Sakuma I, Hattori Y, Kitabatake A (2003) Role of angiotensin II in altered expression of molecules responsible for coronary matrix remodeling in insulin-resistant diabetic rats. Arterioscler Thromb Vasc Biol 23: 2021-2026.

47. Segers VF, Lee RT (2007) Local delivery of proteins and the use of selfassembling peptides. Drug Discov Today 12: 561-568.

48. Zhao Y, Tanaka M, Kinoshita T, Higuchi M, Tan T (2010) Self-assembling peptide nanofiber scaffolds for controlled release governed by gelator design and guest size. J Control Release 147: 392-399.

49. Friedl P (2004) Prespecification and plasticity: Shifting mechanisms of cell migration. Curr Opin Cell Biol 16: 14-23

50. Balaji S (2011) Tissue engineered provisional matrix as a novel approach to enhance diabetic wound healing. Wound Repair and Regeneration in press.

51. Davis ME, Motion JP, Narmoneva DA, Takahashi T, Hakuno D (2005) Injectable self-assembling peptide nanofibers create intramyocardial microenvironments for endothelial cells. Circulation 111: 442-450.

52. Nelson DM, Ma Z, Fujimoto KL, Hashizume R, Wagner WR (2011) Intramyocardial biomaterial injection therapy in the treatment of heart failure: Materials, outcomes and challenges. Acta Biomater 7: 1-15.

53. Dubois G, Segers VF, Bellamy V, Sabbah L, Peyrard S, et al. (2008) Selfassembling peptide nanofibers and skeletal myoblast transplantation in infarcted myocardium. J Biomed Mater Res B Appl Biomater 87: 222-228.

54. Padin-Iruegas ME, Misao Y, Davis ME, Segers VF, Esposito G, et al. (2009) Cardiac progenitor cells and biotinylated insulin-like growth factor-1 nanofibers improve endogenous and exogenous myocardial regeneration after infarction Circulation 120: 876-887.

55. Murcia AM, Hennekens CH, Lamas GA Jiménez-Navarro M, Rouleau JL, et al. (2004) Impact of diabetes on mortality in patients with myocardial infarction and left ventricular dysfunction. Arch Intern Med 164: 2273-2279.

56. Shah AM, Uno H, Køber L, Velazquez EJ, Maggioni AP, et al. (2010) The interrelationship of diabetes and left ventricular systolic function on outcome after high-risk myocardial infarction. Eur J Heart Fail 12: 1229-1237.

57. Falcão-Pires I, Hamdani N, Borbély A, Gavina C, Schalkwijk CG, et al. (2011) Diabetes mellitus worsens diastolic left ventricular dysfunction in aortic stenosis through altered myocardial structure and cardiomyocyte stiffness. Circulation 124: 1151-1159.

58. Heimer R, Bashey RI, Kyle J, Jimenez SA, et al. (1995) TGF- $\beta$ modulates the synthesis of proteoglycans by myocardial fibroblasts in culture. J Mol Cell Cardiol 27: 2191-2198.

59. van Hoeven KH, Factor SM (1990) A comparison of the pathological spectrum of hypertensive, diabetic, and hypertensive-diabetic heart disease. Circulation 82: 848-855.

60. Berg TJ, Snorgaard O, Faber J, Torjesen PA, Hildebrandt P, et al. (1999) Serum levels of advanced glycation end products are associated with left ventricula diastolic function in patients with type 1 diabetes. Diabetes Care 22: 1186-1190.

61. van Heerebeek L, Hamdani N, Handoko ML, Falcao-Pires I, Musters RJ, et al. (2008) Diastolic stiffness of the failing diabetic heart: Importance of fibrosis advanced glycation end products, and myocyte resting tension. Circulation 117 43-51.

62. Banerjee I, Fuseler JW, Price RL, Borg TK, Baudino TA (2007) Determination of cell types and numbers during cardiac development in the neonatal and adult rat and mouse. Am J Physiol Heart Circ Physiol 293: H1883-H1891.

63. Eghbali M (1992) Cardiac fibroblasts: Function, regulation of gene expression, and phenotypic modulation. Basic Res Cardiol 87: 183-189.

64. Porter KE, Turner NA (2009) Cardiac fibroblasts: At the heart of myocardia remodeling. Pharmacol Ther 123: 255-278. 
Citation: Hurley JR, Sheikh AQ, Huang W, Wang Y, Narmoneva DA, et al. (2012) Self Assembling Peptide Nanofibers for Extracellular Matrix Remodeling in Diabetic Cardiomyopathy. J Bioengineer \& Biomedical Sci S5:003. doi:10.4172/2155-9538.S5-003

65. Schröder D, Heger J, Piper HM, Euler G (2006) Angiotensin II stimulates apoptosis via TGF- $\beta 1$ signaling in ventricular cardiomyocytes of rat. J Mol Med (Berl) 84: 975-983.

66. Keogh MB, O'Brien FJ, Daly JS (2010) Substrate stiffness and contractile behaviour modulate the functional maturation of osteoblasts on a collagenGAG scaffold. Acta Biomater 6: 4305-4313.
67. Sieminski AL, Was AS, Kim G, Gong H, Kamm RD (2007) The stiffness of threedimensional ionic self-assembling peptide gels affects the extent of capillarylike network formation. Cell Biochem Biophys 49: 73-83.

68. Narmoneva DA, Vukmirovic R, Davis ME, Kamm RD, et al. (2004) Endothelial cells promote cardiac myocyte survival and spatial reorganization: implications for cardiac regeneration. Circulation 110: 962-968. 